\title{
Aprendizaje basado en problemas en Psicología de las Organizaciones: aplicación de un Ciclo de Mejora
}

\section{Problem-based learning in Organizational Psychology: application of an Improvement Cycle}

ROCíO LÓPEZ-CABRERA

ORCID: https://orcid.org/0000-0002-3915-9129

Universidad de Sevilla. Departamento

de Psicología Social.

mrlopez@us.es

Fecha de recepción:

Fecha de aceptación:

DOI: http://dx.doi.org/10.12795/9788447221912.097

Pp.: 2181-2203 


\section{Resumen}

Con el objetivo de fomentar la adquisición de contenidos por parte del alumnado, situándoles como protagonistas de su propio proceso de aprendizaje, se ha implementado un Ciclo de Mejora en el Aula (CIMA) en la asignatura Psicología de las Organizaciones en el Grado en Psicología (Universidad de Sevilla). En sesiones de grupos reducidos, se ha aplicado una metodología de enseñanza aprendizaje basado en problemas, diseñados en consonancia con la práctica profesional del psicólogo organizacional, y combinando sesiones teóricas y prácticas. Al principio y al final de cada unidad se ha aplicado un cuestionario para evaluar la progresión en las ideas de los alumnos/as, obteniendo resultados positivos tras la aplicación del CIMA. Por último, se discuten diseño, aplicación y evaluación del Ciclo de Mejora y del Modelo Didáctico Personal resultante.

Palabras clave: Psicología de las Organizaciones, Grado en Psicología, docencia universitaria, experimentación docente universitaria, aprendizaje basado en problemas.

\section{Abstract}

With the aim of promoting the acquisition of content by students, placing them as protagonists of their own learning process, an Improvement Cycle has been implemented in the Organizational Psychology Course of the Degree in Psychology (University of Seville). In small group sessions, Problem-based learning methodology was applied, organizing students in small groups and working on problems designed in line with an Organizational Psychologist's professional practice. Complementary theoretical and practical sessions were also implemented. At the beginning and at the end of each unit a questionnaire has been applied to evaluate the progression in the students' ideas, obtaining positive results after the application of the Improvement $\mathrm{Cy}$ cle. Finally, the design, application and evaluation of the Improvement Cycle and the resulting Personal Didactic Model are discussed.

Keywords: "Organizational Psychology", "Psychology Degree", "university teaching", "university teaching experimentation", "problem-based learning". 


\section{Descripción del contexto}

El presente Ciclo de Mejora en el Aula se ha desarrollado en la asignatura Psicología de las Organizaciones, impartida en el segundo curso del Grado en Psicología. Este curso constituye la primera aproximación de los estudiantes a la Psicología en el contexto Organizacional y de los Recursos Humanos, ya que, hasta ese momento, únicamente han trabajado una visión general de la Psicología Social y de la Psicología de los Grupos. El grupo de aplicación de este CIMA ha sido el Grupo 1, con docencia en inglés y castellano, formado por un total de 31 estudiantes, lo que supone una particularidad con respecto a otros grupos de la asignatura, que cuentan con un número de alumnos considerablemente mayor. Esto permite trabajar con un menor número de alumnos/as en las sesiones basadas en aprendizaje basado en problemas, concretamente tres grupos de entre 10 y 12 alumnos/as.

\section{Diseño previo del CIMA}

\section{Mapa de contenidos y problemas}

Para implementar este CIMA se ha desarrollado, en primer lugar, un mapa de contenidos y problemas en el que se refleja tanto los distintos tipos de contenidos a trabajar con los alumnos - conceptuales o "saber", procedimentales o "saber hacer" y actitudinales o "saber ser"- como su interacción (García Díaz, Porlán y Navarro, 2017). Además, se especifican aquellos contenidos considerados estructurales y no estructurales, fomentando una organización que facilitará una visión global de qué queremos trabajar con los estudiantes (Morin 2001). Las Figuras 1 y 2 muestras los mapas de contenido elaborados para las dos unidades temáticas en las que se ha aplicado el CIMA: a) poder e influencia y b) liderazgo. 


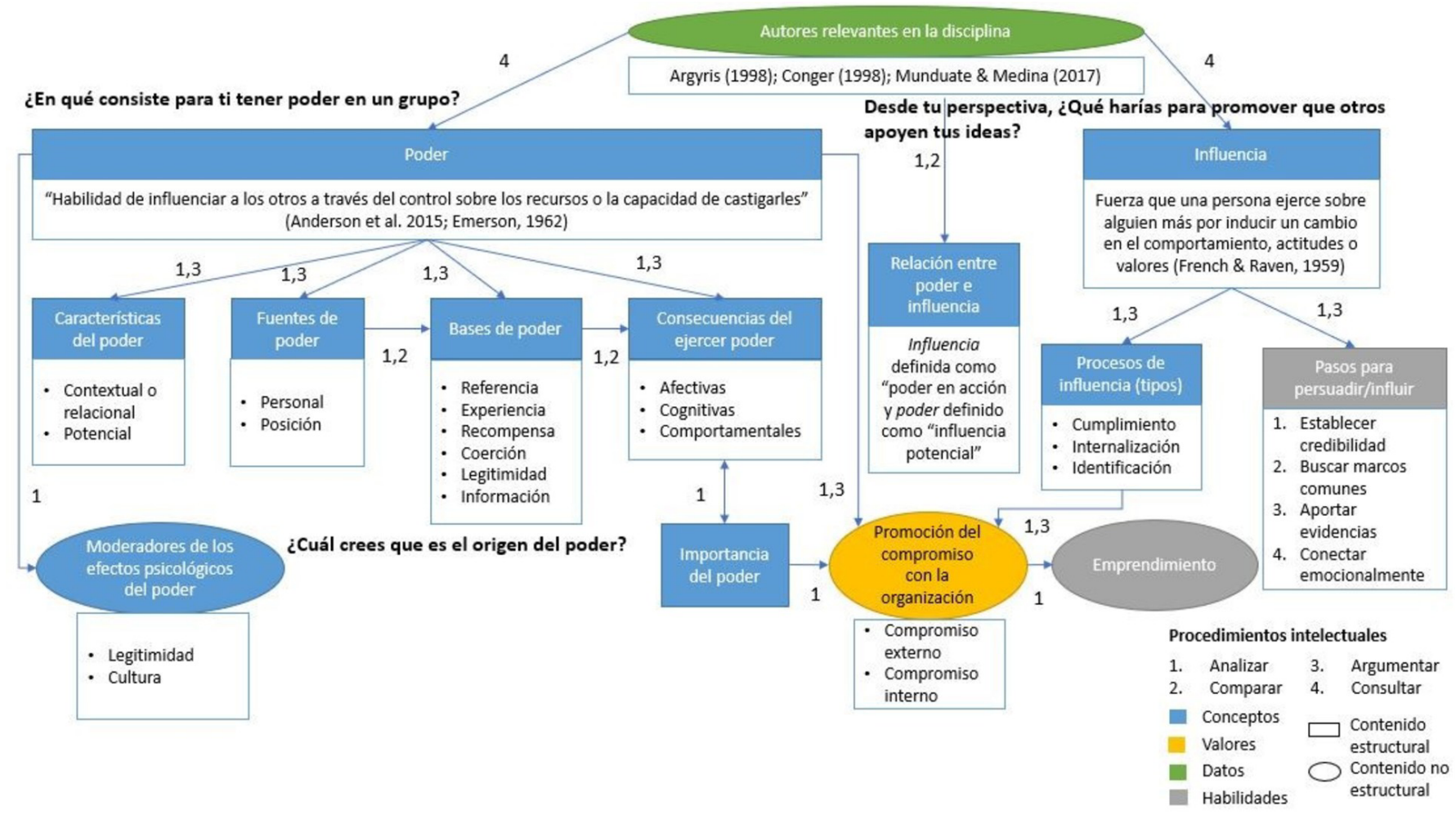

Figura 1. Mapa de contenido para "Poder e influencia"

Jornadas de Formación e Innovación Docente del Profesorado | № 2 (2019) 


\section{Rocío LÓPEZ-CABRERA}

Procedimientos intelectuales
1. Analizar
3. Argument
2. Comparar
4. Consultar
Conceptos
Valores
$\square$ Contenido
Datos estructura
Habilidades
Contenido no estructural

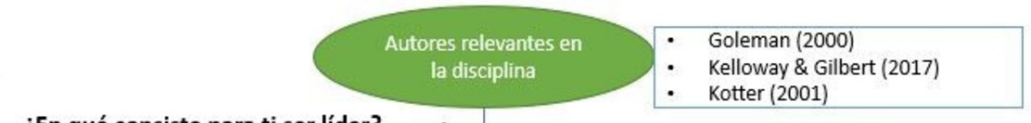

¿En qué consiste para ti ser líder? 4

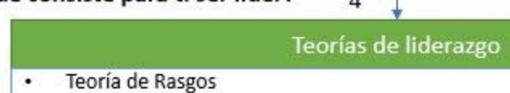

\section{Teorías de}

Teorías Comportame

Teorías de contingencia

Teorías Modernas de Liderazgo (Intercambio L-M; Liderazgo

Transformacional)

- Liderazgo Negativo

\section{¿En qué se diferencia un líder de un gestor? \\ Diferencia entre líder y manager (supervisor) \\ - Los supervisores promueven la estabilidady gestionan la complejidad de la organización. \\ - Los líderes presionan y gestionan el cambio}

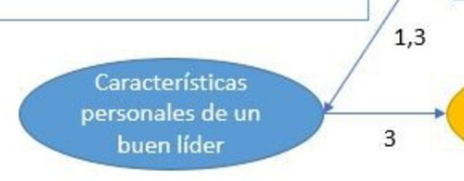

\section{4} 2

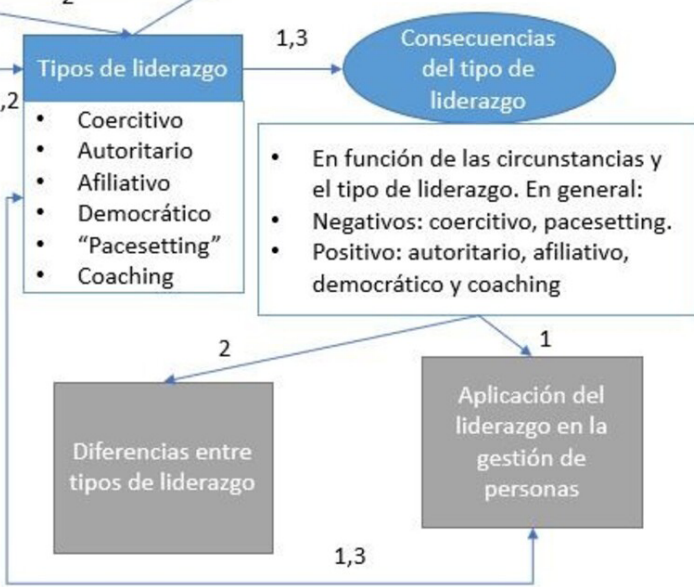

¿Cuál es el liderazgo más efectivo?
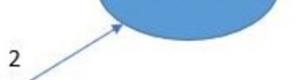
estatus que aporta una

posición o alguien capaz de influir el comportamiento o ideas de los demás.

$1 \uparrow$

Uso de la Inteligencia emocional

- Autoconocimiento

- Autogestión

- Destrezas sociales

- Conocimiento social
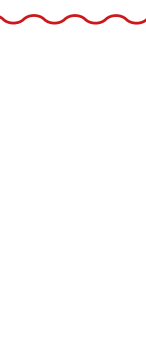

Figura 2. Mapa de contenido y problemas "Liderazgo"

Jornadas de Formación e Innovación Docente del Profesorado | № 2 (2019) 


\section{Modelo metodológico posible argumentado}

El modelo metodológico posible elaborado se basa en el uso de la Metodología basada en Problemas o Problem- Based Learning (PBL por sus siglas en inglés), una metodología de enseñanza universitaria centrada en el estudiante, cuya estructura procura aunar el desarrollo de los conocimientos teóricos del alumnado a la vez que promueve habilidades de trabajo en grupo, pensamiento crítico y razonamiento desde una perspectiva profesional (Sadlo, 2014). La dinámica del Aprendizaje Basado en Problemas consiste en enfrentar a los alumnos a un problema que bien pudiera darse en el contexto profesional, en este caso una organización, en relación con el cual inician un proceso de investigación e indagación para resolverlo y sobre todo aprender los conceptos que de él se derivan. Actualmente el entorno laboral exige a los futuros profesionales haber adquirido no sólo conocimientos teóricos y prácticos específicos sino también determinadas competencias como el trabajo en grupo, habilidades de comunicación o autogestión e independencia (Dueñas, 2001; Savin-Baden, 2003). Por tanto, este modelo fomenta el aprendizaje de contenidos tanto conceptuales como actitudinales y también procedimentales (García Díaz y col., 2017), lo cual es, como se ha hecho referencia anteriormente, uno de los objetivos de este CIMA.

En definitiva, el aprendizaje basado en problemas consiste en someter a los alumnos a una simulación del proceso real de ejercicio de la profesión: ante un problema o caso relacionado con el contenido de la asignatura y con la práctica profesional, se trabaja en equipo para alcanzar un nivel óptimo de comprensión del fenómeno y plantear objetivos de aprendizaje referentes a la materia, y también de forma individual, poniéndose en común con el resto del equipo las idea personales para dar respuesta a las incógnitas planteadas. De esta manera, el estudiante es el principal agente de su propio aprendizaje y el profesor 
se convierte en facilitador del proceso; son los alumnos los que construyen su propio conocimiento de forma más autónoma y activa a la vez que se activan competencias como el trabajo en grupo, el liderazgo o la solución de problemas (Maudsley, 1999; Schultz-Ross y Kline, 1999).

Concretamente, para el desarrollo de este CIMA se han trabajado dos casos o problemas en cuatro sesiones, dedicando un total de cuatro horas a cada uno, hasta completar las ocho horas del ciclo. Estos casos han sido leídos previamente por los/las estudiantes de manera que ya han tenido una primera aproximación al inicio de la primera sesión. Tal y como se muestra en el mapa de contenidos, en base a cada problema se han trabajado tres subproblemas o preguntas, a las que los/las alumnos/as llegarán de forma autónoma mediante la discusión del caso, de sus ideas previas y de las intervenciones esporádicas del docente en las actividades de contraste. La Figura 3 muestra el Modelo Metodológico posible diseñado para este CIMA.

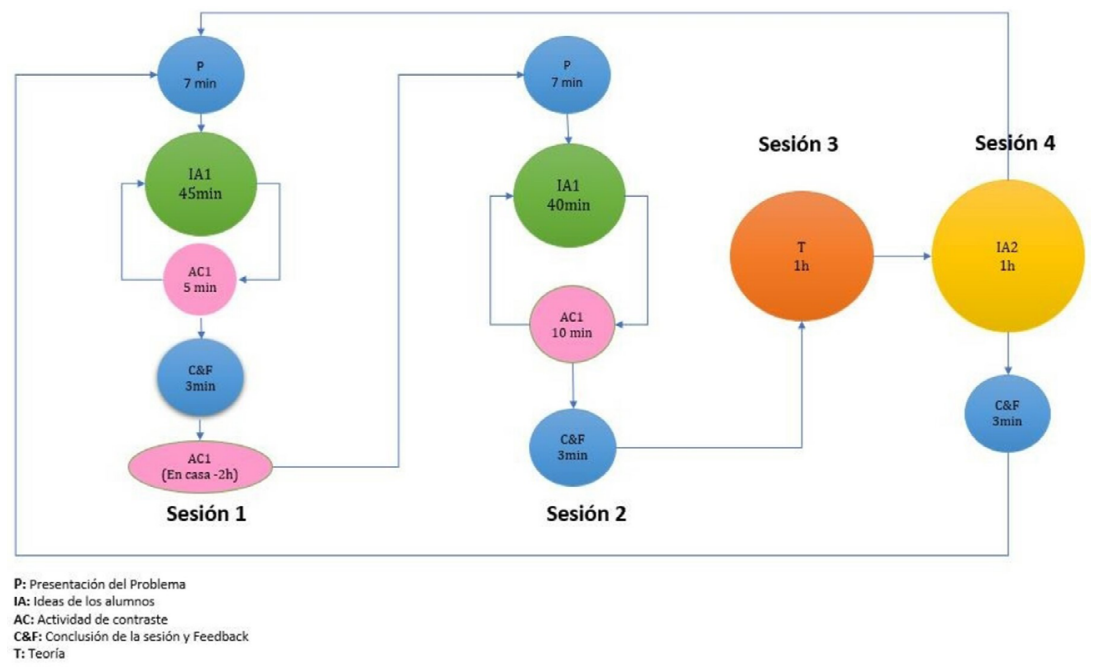

Figura 3. Modelo metodológico posible del CIMA.

Jornadas de Formación e Innovación Docente del Profesorado | № 2 (2019) Esta obra se distribuye con la licencia Creative Commons Reconocimiento-NoComercial-SinObraDerivada 


\section{Secuencia de actividades detallada}

Para cada una de las sesiones desarrolladas en este CIMA se ha programado la correspondiente secuencia de actividades en función de los dos casos tratados. A su vez, los subproblemas reflejados en los mapas de contenido han vertebrado las dos primeras sesiones, sirviendo como guía también en las dos últimas. En la Tabla 1 se presenta la secuencia de actividades detallada y enumerada.

Como se ha indicado anteriormente, esta secuencia se ha llevado a cabo para cada caso relativo a las dos unidades temáticas: a) poder e influencia y b) liderazgo, completando las ocho horas de duración del CIMA.

Tabla 1. Secuencia de actividades detallada.

\begin{tabular}{|l|l|l|l|}
\hline \multicolumn{2}{|l|}{ SESIÓN 1. Presentación del Caso } & Tiempo \\
\hline Fase & Actividad & Descripción & 7 min \\
\hline P & Presentación & $\begin{array}{l}\text { Presentación del problema/caso en el } \\
\text { contexto de las organizaciones. Le explico } \\
\text { a los estudiantes que esta situación puede } \\
\text { darse en desempeño profesional de como } \\
\text { psicólogo/a organizacional al tener que } \\
\text { gestionar la entrada en un nuevo contexto } \\
\text { de trabajo. Además, se asignan los roles a } \\
\text { desempeñar durante la sesión: “líder de la } \\
\text { discusión" y "secretario/a). Así se fomenta } \\
\text { que los estudiantes tomen responsabilidad } \\
\text { de su proceso de aprendizaje, empezando } \\
\text { por coordinar la sesión. De esta forma } \\
\text { adaptan el ritmo y la forma de organizar } \\
\text { los contenidos de la forma que mejor se } \\
\text { adapte a sus necesidades. Los roles rotan } \\
\text { semanalmente y suelen asignarse de forma } \\
\text { voluntaria entre los estudiantes, en caso } \\
\text { de que no haya consenso elijo yo entre los } \\
\text { estudiantes que aún no han ejercido esos } \\
\text { roles. }\end{array}$ & \\
\hline
\end{tabular}

Jornadas de Formación e Innovación Docente del Profesorado | № 2 (2019) Esta obra se distribuye con la licencia Creative Commons Reconocimiento-NoComercial-SinObraDerivada Internacional (CC BY-NC-ND 4.0.) 


\begin{tabular}{|c|c|c|c|}
\hline IA1 & $\begin{array}{l}\text { Análisis del } \\
\text { problema }\end{array}$ & $\begin{array}{l}\text { Se presenta un problema o caso práctico } \\
\text { que pueda presentarse en el del ejercicio } \\
\text { profesional de la Psicología de las } \\
\text { Organizaciones, que los alumnos ya han } \\
\text { leído como preparación para la sesión. En } \\
\text { este CIMA, además como primer análisis de } \\
\text { la temática del caso, los/las estudiantes } \\
\text { responderán al cuestionario de diagnóstico } \\
\text { inicial. Además, dado que en esta actividad se } \\
\text { exploran las ideas previas de los estudiantes } \\
\text { sobre el tema que se está trabajando, en el } \\
\text { caso de este ciclo de mejora el poder y la } \\
\text { influencia en las organizaciones, se llevarán } \\
\text { a cabo las siguientes tareas: } \\
\text { Tarea 1) Clarificación de conceptos poco } \\
\text { claros: } \\
\text { El problema que los/las estudiantes } \\
\text { tienen que trabajar en los seminarios } \\
\text { pueden incluir términos que pueden } \\
\text { interpretarse de distintas maneras o que } \\
\text { no todos comprenden; por lo tanto, deben } \\
\text { consensuarse y aclararse antes de que } \\
\text { pueda iniciarse la discusión de forma } \\
\text { apropiada. Cuando hay dudas, los/las } \\
\text { estudiantes las resuelven entre ellos, si no } \\
\text { es posible, intervendría para ayudarles con } \\
\text { preguntas que les hagan reflexionar sobre } \\
\text { partes concretas del caso. } \\
\text { Tarea 2) Definición del problema: } \\
\text { Es importante alcanzar un acuerdo entre } \\
\text { todos los miembros del grupo sobre cuál es } \\
\text { el tema que refleja el problema, ya que esto } \\
\text { determinará el resto de la discusión. }\end{array}$ & $40 \mathrm{~min}$ \\
\hline
\end{tabular}


Mediante la técnica "brainstorming", todas las ideas que surgen de manera libre durante la discusión son anotadas por el secretario y discutidas por el grupo; estas ideas pueden contribuir a una mayor comprensión de alguna de las explicaciones del problema o caso en cuestión. Esta tarea, parte de la actividad basada en ideas de los alumnos se alterna con la siguiente actividad de contraste, en la que, con pequeñas intervenciones cuando se requieren como docente intervengo en la discusión con preguntas sobre el caso. Mi rol en esta actividad por tanto es supervisar que la discusión se desarrolle con normalidad y que lleguen a definir y analizar el problema correctamente. Esto conlleva incentivar con preguntas la conversación Ej. "¿qué pensáis? ¿de qué creéis que trata el problema?" y animar a los estudiantes a participar.

Tarea 4) Listado y profundización de ideas principales profundizar En primer lugar, todas las ideas de la en las explicaciones del caso fase anterior se agrupan y se discuten ampliamente. En esta fase el profesorado interviene centrando el debate con preguntas que los lleven a analizar en profundidad el caso planteado y se comparan con los conocimientos previos de los estudiantes sobre las teorías formuladas en la literatura.

Tarea 5) Formular los objetivos de aprendizaje

Se fijan los objetivos de aprendizaje en base a las ideas esbozadas en el paso 4. Los objetivos de aprendizaje son las tareas que se trabajarán en la fase de estudio individual al estudiar la literatura. Estas tareas no se dividen entre los alumnos, todos deben trabajar todos los objetivos de aprendizaje elaborados. 


\begin{tabular}{|c|c|c|c|}
\hline$C \& F$ & Conclusión & $\begin{array}{l}\text { El líder de la discusión sintetiza las ideas } \\
\text { discutidas en la discusión del problema. } \\
\text { Por mi parte, doy feedback de la sesión } \\
\text { particularmente al líder de la discusión y al } \\
\text { secretario/a y cierro la sesión, recordando } \\
\text { las lecturas que deben realizar y cuál será la } \\
\text { dinámica de la próxima sesión. }\end{array}$ & $3 \mathrm{~min}$ \\
\hline $\mathrm{AC1}$ & $\begin{array}{l}\text { Revisión de } \\
\text { la literatura } \\
\text { relevante } \\
\text { (horario no } \\
\text { lectivo) }\end{array}$ & $\begin{array}{l}\text { Tarea 6) Lectura de artículos científicos } \\
\text { Cada alumno/a estudia la literatura } \\
\text { propuesta y si lo requieren los objetivos de } \\
\text { aprendizaje amplían con recursos científicos } \\
\text { que exponen a sus compañeros. } \\
\text { Durante la sesión propongo que de las } \\
\text { lecturas optativas se distribuyan entre } \\
\text { los miembros del grupo y que cada uno } \\
\text { elabore un resumen que se comparta con } \\
\text { los compañeros/as, para que todo el mundo } \\
\text { conozca el contenido de todo el material. } \\
\text { Los materiales obligatorios se trabajarán } \\
\text { individualmente. }\end{array}$ & $120 \mathrm{~min}$ \\
\hline \multicolumn{4}{|c|}{ SESIÓN 2. Discusión del Problema en base a la literatura } \\
\hline & Actividad & Descripción & Tiempo \\
\hline P & $\begin{array}{l}\text { Presentación } \\
\text { del problema }\end{array}$ & $\begin{array}{l}\text { Introduzco la nueva sesión brevemente, } \\
\text { haciendo referencia a la sesión anterior } \\
\text { y a la temática del problema. Se asignan } \\
\text { los roles a desempeñar durante la sesión: } \\
\text { "líder de la discusión" y "secretario/a). El } \\
\text { secretario/a de la sesión anterior ha sido } \\
\text { el líder de la discusión y se ha ofrecido un } \\
\text { estudiante como voluntario para el rol de } \\
\text { secretario, anotando las ideas principales } \\
\text { de las lecturas que dan respuesta a los } \\
\text { objetivos de aprendizaje. }\end{array}$ & $10 \mathrm{~min}$ \\
\hline IA1 & $\begin{array}{l}\text { Respuesta } \\
\text { a los } \\
\text { objetivos de } \\
\text { aprendizaje }\end{array}$ & $\begin{array}{l}\text { Los/las estudiantes dan respuesta a los } \\
\text { objetivos de aprendizaje planteados en la } \\
\text { sesión anterior, explicando el contenido } \\
\text { de las lecturas que han leído, discutiendo } \\
\text { además con sus compañeros/as las } \\
\text { respuestas a los objetivos de aprendizaje, } \\
\text { llegando a una solución consensuada. }\end{array}$ & $40 \mathrm{~min}$ \\
\hline
\end{tabular}




\begin{tabular}{|c|c|c|c|}
\hline $\mathrm{AC1}$ & $\begin{array}{l}\text { Discusión de } \\
\text { la literatura } \\
\text { revisada }\end{array}$ & $\begin{array}{l}\text { Además de responder a las preguntas } \\
\text { concretas planteadas, los/las estudiantes } \\
\text { completan el mapa conceptual con } \\
\text { información adicional que han aprendido } \\
\text { revisando el material. Esta fase se alterna } \\
\text { con la anterior para cada objetivo de } \\
\text { aprendizaje. }\end{array}$ & $10 \mathrm{~min}$ \\
\hline \multicolumn{4}{|c|}{ SESIÓN 3. Sesión conceptual } \\
\hline & Actividad & Descripción & Tiempo \\
\hline $\mathrm{T}$ & $\begin{array}{l}\text { Presentación } \\
\text { de contenidos } \\
\text { y dudas }\end{array}$ & $\begin{array}{l}\text { Trabajo de los conceptos teóricos por parte } \\
\text { del profesorado, conectando los mismos } \\
\text { con los debates previos pero unificado las } \\
\text { ideas de los alumnos en cuanto a la teoría. }\end{array}$ & $50 \mathrm{~min}$ \\
\hline \multicolumn{4}{|c|}{ SESIÓN 4. Resolución del caso } \\
\hline & Actividad & Descripción & Tiempo \\
\hline \multirow[t]{3}{*}{ IA2 } & Cuestionario & $\begin{array}{l}\text { Los/las estudiantes completan de nuevo el } \\
\text { cuestionario de diagnóstico inicial }\end{array}$ & $15 \mathrm{~min}$ \\
\hline & $\begin{array}{l}\text { Resolución } \\
\text { del Problema: } \\
\text { Aplicación de } \\
\text { lo aprendido }\end{array}$ & $\begin{array}{l}\text { Durante esta sesión, por grupos, los } \\
\text { estudiantes relacionan los aspectos del } \\
\text { caso inicial con los conceptos principales } \\
\text { de la literatura. De esta manera toman } \\
\text { consciencia de lo aprendido sobre la } \\
\text { temática de la unidad. }\end{array}$ & $35 \mathrm{~min}$ \\
\hline & $\begin{array}{l}\text { Exposición } \\
\text { de las } \\
\text { propuestas }\end{array}$ & $\begin{array}{l}\text { Los grupos exponen brevemente a sus } \\
\text { compañeros sus soluciones al caso } \\
\text { trabajado y se debaten entre todos. }\end{array}$ & $5 \mathrm{~min}$ \\
\hline$C \& F$ & $\begin{array}{l}\text { Conclusiones } \\
\text { y Feedback }\end{array}$ & $\begin{array}{l}\text { Breves comentarios por parte del } \\
\text { profesorado sobre las presentaciones de los } \\
\text { alumnos de su análisis del caso. }\end{array}$ & $5 \mathrm{~min}$ \\
\hline
\end{tabular}

\section{Cuestionarios inicial-final}

Como parte de la primera y de la última actividad de ideas previas, los/las alumnos/as respondieron a un cuestionario inicial-final, con el objetivo de hacer un seguimiento de su evolución en relación con los subproblemas planteados. Dichas preguntas han sido: 
Caso: ¿Quién será la estrella del equipo?

1. ¿En qué consiste para ti tener poder en un grupo?

2. En base a tu experiencia, ¿Cuál dirías que es el origen del poder?

3. Desde tu perspectiva, ¿Qué harías para promover que otros apoyen tus ideas?

Caso: Ayuda, necesitamos un buen líder

1. ¿En qué consiste para ti ser líder?

2. ¿Cuál es el liderazgo más efectivo?

3. ¿En qué se diferencia un líder de un gestor?

\section{Aplicación del CIMA}

Durante la aplicación del CIMA las sesiones se desarrollaron según se describen a continuación:

Caso/Problema: Poder e influencia

Sesión 1: Análisis del caso y planteamiento de los problemas u objetivos de aprendizaje.

De manera previa a la clase, se puso a disposición de los estudiantes en la Enseñanza Virtual el caso práctico para su lectura: ¿Quién será la estrella del equipo?

En la primera sesión se realizó la presentación del caso práctico y del problema que puede tener lugar en el contexto de las organizaciones: un equipo de trabajo multidisciplinar, que se ha creado para llevar a cabo un proyecto en el ámbito de una empresa textil. Los miembros del equipo, residentes en distintas localizaciones, consideran esta una oportunidad para destacar en su puesto de trabajo, con idea de conseguir un posible ascenso. En estas circunstancias todos quieren llegar a ser la estrella del equipo.

Al iniciar la sesión, se asignaron los roles principales ("líder de la discusión" y "secretario/a") que coordinaron la sesión. En el caso del secretario, un alumno de cada uno 
de los grupos asumió el rol voluntariamente. El líder de la discusión lo asumieron los estudiantes que habían sido secretarios la sesión anterior. Como tarea inicial, los/las estudiantes cumplimentaron el cuestionario inicial. Para ello, les expliqué que como parte del Curso en Formación Docente que estaba realizando, iban a participar en un ciclo de mejora en la asignatura. Así, el objetivo de pedirles que cumplimentaran este cuestionario anónimo era conocer sus ideas previas sobre el contenido de la unidad, sin un punto de vista académico, únicamente teniendo en consideración sus opiniones o experiencias. La respuesta de los estudiantes fue positiva y respondieron a las tres preguntas planteadas de forma extensa. Una vez finalizado el cuestionario (15/20 min. aproximadamente) se inició el análisis del caso.

En primer lugar, se clarificaron los términos y conceptos que suscitaban dudas. En este caso hubo necesidad de clarificar términos en inglés, que los propios alumnos/as resolvieron entre ellos sin intervención del docente. A continuación, los estudiantes llegaron a un acuerdo en cuanto a la definición el problema, estableciendo en una frase de qué trata el caso. Esta definición se consensuó de manera conjunta, determinando el transcurso de la sesión. Para analizar el problema, los estudiantes utilizaron la técnica "brainstorming". Todas las ideas que surgieron en el análisis fueron anotadas y organizadas por el secretario para contribuir a una mayor comprensión del caso. Durante el análisis intervine en varios momentos para ayudar a los estudiantes a profundizar en qué es el poder y qué consecuencias tiene, así como en la diferencia de comportamiento de un líder. Con mis intervenciones a base de preguntas, ellos fueron capaces de indagar en sus ideas previas para diferenciar ambos conceptos. Mi rol en esta actividad ha sido por tanto supervisar que la discusión se desarrollara con normalidad y definieran y analizaran el problema correctamente con el uso de preguntas ( Ej. "¿qué pensáis?, ¿de qué creéis que trata el problema?"...) que les ayuden a cuestionarse cómo avanzar y animarlos 
a participar (Finkel, 2008). Finalmente, los estudiantes fijaron sus objetivos de aprendizaje en base a las ideas esbozadas en la actividad anterior. Los objetivos de aprendizaje son las tareas que se trabajarán en la fase de estudio individual al traabjar la literatura. Estas tareas no se dividen entre los alumnos, todos deben trabajar todos los objetivos de aprendizaje elaborados. Para finalizar, el líder de la discusión sintetizó las ideas discutidas en la discusión del problema. Por mi parte, di a los alumnos feedback de la sesión, particularmente al líder de la discusión y al secretario/a, y cierro la sesión, recordando las lecturas que debían realizar e informado cuál sería la dinámica de la próxima sesión.

Mi experiencia en esta sesión del CIMA ha sido muy positiva, ya que los alumnos han participado activamente, lo que me ha permitido no tener que intervenir salvo en contadas ocasiones. A pesar de que no intervenir más a menudo es dificil, el tener muy marcados los pasos de la sesión, facilitó la tarea de los alumnos/as y les proporcionó la seguridad para participar de forma más autónoma. Aun así, hay estudiantes a los que les cuesta participar por temor a equivocarse o simplemente por timidez, a lo que también se suma que las clases sean en inglés, lo que dificulta que los menos proactivos participen. Así, cuando alguno ha tenido la necesidad de intervenir en castellano, le he animado a hacerlo, tras lo cual yo lo he reformulado en inglés y han continuado la sesión.

Sesión 2: Discusión de las lecturas para resolver los objetivos de aprendizaje.

Durante esta actividad los estudiantes explicaron el contenido de las lecturas leídas y respondieron a los objetivos de aprendizaje, llegando a una solución consensuada. La sesión se ha desarrollado normalmente, retomando la discusión de la sesión anterior, en base a las ideas generales. Durante esta sesión, llevamos a cabo la observación con otro compañero del curso de Docencia. Los estudiantes han sido muy participativos, a excepción 
de una alumna, todos han contribuido a debatir las lecturas y responder a los objetivos de aprendizaje. Ha sido interesante ver cómo han cambiado algunas de sus conceptualizaciones una vez que han leído sobre el tema y han añadido contenido teórico como fundamento a sus nuevas ideas. En esta sesión ha sido determinante la actuación de la líder del grupo, que ha motivado la participación de los demás.

Sesión 3: Sesión conceptual "Poder e influencia"

En esta sesión hemos trabajado los conceptos teóricos. El propósito era sido unificar las ideas teóricas de todos los grupos y afianzar los conceptos. Con esta sesión he intentado que los estudiantes tengan un espacio para contrastar sus ideas no sólo con sus compañeros de grupo sino con todos los demás y asegurarse de que lo que han aprendido es adecuadoí. Después de varias sesiones muy proactivas, tenía algunas dudas sobre como funcionaría una sesión teórica. Sin embargo, la dinámica ha continuado con la misma línea que la de los grupos de PBL. Los estudiantes han hecho preguntas, han hecho referencia a lo que han leído y debatido previamente y se han mostrado motivados, lo que ha me ha facilitado a mí también llevar la clase de forma más dinámica de lo que cabría esperar de una sesión teórica.

Sesión 4: Aplicación de los conceptos aprendidos en la resolución del caso práctico.

Durante esta sesión, por grupos, los estudiantes relacionaron los aspectos del caso inicial con los conceptos principales de la literatura. De esta manera toman consciencia de lo aprendido sobre la temática de la unidad. Como primera actividad, los/las estudiantes completaron de nuevo el cuestionario de diagnóstico inicial (final en este caso). Posteriormente, los grupos expusieron brevemente a sus compañeros las soluciones al caso trabajado y se debatieron entre todos. En esta sesión los alumnos han trabajado en grupo para proponer soluciones al caso 
en base a la literatura y han elaborado un documento que han expuesto a sus compañeros y me han entregado para control de asistencia y evaluación. En esta sesión los estudiantes han podido de alguna manera plasmar lo que han aprendido en una forma similar al trabajo de un psicólogo/a organizacional. Tras analizar de nuevo el problema, y exponer los resultados, realicé breves comentarios sobre las presentaciones de los alumnos sobre su análisis del caso. Esta sesión ha sido muy interesante, ya que nos ha permitido ver la aplicación práctica de la teoría que hemos estado trabajando durante las sesiones anteriores. En mi opinión, creo que verse en el papel de un profesional les ha sido de gran utilidad.

\section{Caso/Problema: Liderazgo}

Sesión 1: Análisis del caso y planteamiento de los problemas u objetivos de aprendizaje.

En esta sesión se ha seguido la misma dinámica que en la sesión 1 anterior. Los estudiantes también han respondido al cuestionario de ideas iniciales antes de analizar el problema y crear los objetivos de aprendizaje. En esta sesión los estudiantes han sido muy autónomos y apenas han necesitado de mis intervenciones. Es gratificante ver como los alumnos/as evolucionan con el paso de las semanas tomando las riendas de su propio proceso de aprendizaje.

Sesión 2: Discusión de las lecturas para resolver los objetivos de aprendizaje.

Durante esta sesión se ha seguido la misma dinámica de la sesión 2 del tema anterior. Se ha desarrollado con normalidad, con los estudiantes participando proactivamente en las sesiones y respondiendo a los objetivos de aprendizaje en base a las lecturas, con intervenciones breves por mi parte, particularmente centradas en clarificar algunas teorías de liderazgo y fomentar su participación. 
Sesión 3: Sesión conceptual "Liderazgo"

Trabajo de los conceptos teóricos por parte del profesorado, conectando los mismos con los debates previos pero unificado las ideas de los alumnos en cuanto a la teoría. Tal y como se describe en la sesión anterior la sesión se ha desarrollado de forma satisfactoria, con los alumnos/as participando de forma activa, a pesar de ser una clase teórica.

Sesión 4: Aplicación de los conceptos aprendidos en la resolución del caso práctico.

En la última sesión del CIMA, hemos vuelto a tener una sesión de aplicación de los conceptos aprendidos al problema, en este caso sobre liderazgo. Los/las estudiantes han manifestado el interés que les despertaron estas sesiones en las que pudieron ver claramente su progreso desde la primera vez que intentaron analizar el problema hasta la segunda en la que ya habían revisado el contenido. Para mí como docente estos avances también quedan patentes y ponen de manifiesto la eficacia no sólo del aprendizaje de contenidos conceptuales sino también procedimentales y actitudinales.

Durante la aplicación del CIMA se ha llevado a cabo un cuestionario de ideas previas para evaluar los modelos mentales de los estudiantes al inicio de cada caso práctico. En primer lugar, se les presentó a los alumnos/as con antelación el caso práctico para que lo leyeran previamente. Tras explicarles el objetivo del Curso General de Docencia Universitaria y de la actividad concreta, se les pidió que cumplimentaran un breve cuestionario anónimo, de tres preguntas o subproblemas en base a su conocimiento o ideas previas sobre el tema. Como constatación de los buenos resultados del CIMA, a continuación, de forma representativa, se muestra la escalera de aprendizaje (Rivero y Porlán, 2017) obtenida a partir de los resultados de los cuestionarios inicial-final para el primer subproblema del caso sobre "Poder e Influencia": ¿En qué consiste para ti tener poder en un grupo? (Figura 4). 


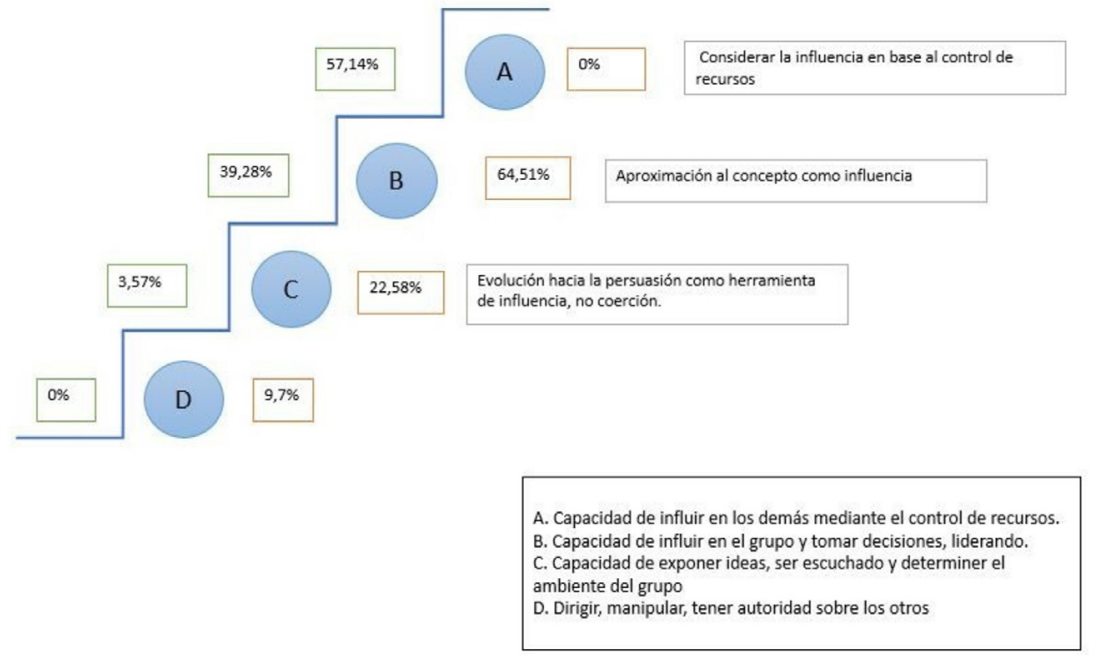

Figura 4. Escalera de aprendizaje subproblema 1 (Problema 1: Poder e influencia)

En esta pregunta se puede comprobar que hay un avance significativo en los modelos mentales de los/las alumnos/as. En el cuestionario inicial, ninguno de ellos dio una respuesta que abarcara plenamente el concepto, y de hecho había alumnos que confundían el tener poder con la coacción. Sin embargo, los resultados del cuestionario final indican que la mayoría de los estudiantes han alcanzado el punto más alto de la escalera, no quedando ninguno en el más inferior. De hecho, el porcentaje en los escalones intermedios ha disminuido considerablemente en el cuestionario final.

En general, los datos obtenidos demuestran la eficacia del CIMA, dado el avance de los estudiantes en cuanto a sus modelos mentales, así como la utilidad de usar este tipo de herramientas para orientar la docencia. En el futuro, me inclino a volver a utilizar este tipo de evaluación de ideas previas y finales del alumnado, tanto para el diagnóstico de las ideas de los alumnos como de eficacia de la docencia. 


\section{Evaluación del CIMA}

El CIMA descrito ha funcionado, en general, muy satisfactoriamente. A este respecto, como elementos a mantener en futuros ciclos destacaría varias cuestiones. En primer lugar el uso del cuestionario inicial para evaluar las ideas previas de los alumnos. El uso de este instrumento da una perspectiva muy clara con la que trabajar para apoyar el aprendizaje de los estudiantes, maximizando el rendimiento, ya que es posible usarlas tanto para mostrarles su propio progreso como para conocer dónde hay que prestar mayor atención como docente. A este respecto, el trabajar con casos o problemas ha facilitado que los alumnos/as vayan construyendo su aprendizaje no sólo en base a sus propias ideas sino contextualizándolas en su práctica profesional futura. Esto les da una perspectiva de por qué es relevante lo que estudian, de la que carece un sistema de enseña más tradicional. Finalmente, la elaboración y el uso como guía del mapa de contenidos me ha facilitado mucho la labor de coordinación de los tutoriales, ya que me ha permitido hacer un seguimiento de los contenidos que debían tratarse en clase de forma organizada.

Los resultados han sido buenos y la experiencia, tanto para los estudiantes como para mí como docente ha sido positiva y enriquecedora. No obstante, cabe destacar que hay estudiantes que se sienten menos cómodos en este tipo de metodologías que requieren de su participación expresa y continuada, y para ellos el CIMA ha supuesto algunas dificultades. Sin embargo, he intentado que lo tomaran como un reto y una oportunidad de mejorar en competencias, no sólo en aprendizaje de conceptos lo que les ha ayudado a ir mejorando paulatinamente en el transcurso del CIMA.

Como mejora en próximos CIMA, creo que sería beneficioso probar nuevos casos en los que basar los 
subproblemas. Los utilizados este año han funcionado, pero en ciertos momentos han requerido de mi intervención para evitar que los estudiantes se desviaran de la temática en exceso. Si bien es cierto que, dado que son casos prácticos, conforme van avanzando en la materia son capaces de interconectar los temas, el uso de casos prácticos que acoten más los problemas a tratar en cada unidad puede beneficiar el desarrollo de los CIMA.

\section{Modelo Didáctico Personal: Principios didácticos argumentados}

Esta experiencia me ha permitido aportar un marco teórico y procedimental a mi docencia, la cual hasta ahora impartía basada en mi propia experiencia como alumna, intentando subsanar errores o implementar mejoras que detecté desde la perspectiva de estudiante. Sin embargo, he aprendido a sistematizar dichos cambios y en general a desarrollar un Modelo Didáctico Personal. En cuanto al aprendizaje de los/as alumnos/as una de las ideas clave que me quedo es la importancia de considerar al alumnado protagonista de su propio aprendizaje y por lo tanto centrar en ellos el proceso de enseñanza. Esto implica en primer lugar, asumir como docentes un rol secundario de acompañamiento de los/las alumnos/as en su desarrollo y no a marcar el camino que nosotros consideramos. A este respecto, la importancia de trabajar con los modelos mentales de los estudiantes como base de nuestra docencia es clave, y nosotros tenemos que ser flexibles y adecuar nuestra labor para maximizar los beneficios que en términos de conocimientos conceptuales, actitudinales y procedimentales nuestros estudiantes puedan adquirir. En cuanto al contenido de enseñanza a impartir, considero muy necesario elaborar un mapa de contenido para en primer lugar tener organizados los contenidos conceptuales, procedimentales y actitudinales a impartir y cómo se relacionan, así como la conexión con los problemas. Asimismo, 
esta estructura permite priorizar contenidos estructurales sin perder de vista la imagen general del tema tratado, lo que es muy útil al trabajar con problemas en los que es fácil que los/las estudiantes se dispersen y somos nosotros los que tenemos que asegurarnos de guiar su aprendizaje.

En este sentido, tiene también mucha relevancia a la hora de estructurar nuestra docencia, el diseño y elaboración previa del modelo metodológico personal. Me ha parecido muy útil ser capaz de dar nombre a distintas tareas que ya llevaba a cabo en el aula, pero a las que aún no había ubicado en un modelo metodológico. En este sentido la creación de las fases del modelo, aunque resulte laboriosa, permite organizar qué queremos hacer en el aula. Con relación a esto, crear la secuencia de actividades concreta para cada fase, contribuye a fijar las sesiones y es algo que creo que incorporaré a mi docencia habitual.

Finalmente, en cuanto a la evaluación, para mí ha sido reconfortante ver el avance generalizado del alumnado en las escaleras de aprendizaje y poder ver plasmado su cambio en las ideas previas y finales. Aunque no lo considero parte de la evaluación continua, sí como herramienta de diagnóstico para orientar las sesiones. En este sentido sí que considero que, si centramos la docencia en el alumnado, la participación debe ser evaluada, ya sea con un portafolio o con una rúbrica. En mi caso, además de las pruebas de evaluación continua, sistematizar qué se espera de la participación del alumnado en tareas concretas creo que ha resultado muy útil, tanto a ellos para saber dónde mejorar, como a mí para evaluarlos. 


\section{Referencias}

Finkel, D. (2008). Dar clase con la boca cerrada. Valencia, España: Universitat de València.

García-Díaz, E., Porlán, R. y Navarro, E. (2017). Los fines y los contenidos de enseñanza. En R. Porlán (Coord.), Enseñanza Universitaria. Cómo mejorarla (pp. 55-72). Madrid, España: Morata.

Maudsley, G. (1999). Roles and responsibilities of the problem-based learning tutor in the undergraduate medical curriculum. British Medical Journal, 318, 657-660.

Morin, E. (2001). La mente bien ordenada. Repensar la reforma. Reformar el pensamiento. Barcelona. Seix Barral. Rivero, A. y Porlán, R. (2017). La evaluación en la enseñanza universitaria. En R. Porlán (Coord.), Enseñanza Universitaria. Cómo mejorarla (pp. 73-91). Madrid, España: Morata.

Sadlo, G. (2014). Using problem-based learning during student placement to embed theory in practice. The Higher Education Academy, 2 (1), 6-19.

Savin-Baden, M. (2003). Facilitating Problem-Based Learning: Illuminating Perspectives. The Society for Research into Higher Education \& Open University Press. Schultz-Ross, R.A., y Kline, A.E. (1999). Using problem-based learning to teach forensic psychiatry. Academic Psychiatry, 23, 37-41.

Jornadas de Formación e Innovación Docente del Profesorado | № 2 (2019) Esta obra se distribuye con la licencia Creative Commons Reconocimiento-NoComercial-SinObraDerivada 4.0 Internacional (CC BY-NC-ND 4.0.) 